\title{
Empowering Living Matter and the Fever Effect of Global Warming
}

\author{
Simon Berkovich* \\ Emeritus Professor of Engineering and Applied Science, The George Washington University, USA
}

*Corresponding author: Simon Berkovich, Emeritus Professor of Engineering and Applied Science, The George Washington University, Washington, DC, 20052, USA, E-mail: berkov@gwu.edu

Received: 02 Aug, 2019 | Accepted: 02 Sep, 2019 | Published: 09 Sep, 2019

Citation: Berkovich S (2019) Empowering Living Matter and the Fever Effect of Global Warming. J Neurol Neurobiol 6(1): dx.doi.org/10.16966/23797150.157

Copyright: () 2019 Berkovich S. This is an open-access article distributed under the terms of the Creative Commons Attribution License, which permits unrestricted use, distribution, and reproduction in any medium, provided the original author and source are credited.

The amount of mechanical energy being produced by the muscles of all animals is so far several times more than the energy from all thermal engines created by humans. But what is most wonderful, and we have to confess - up to nowadays scientists do not understand the essence of the muscle process. -P. L. Kapitsa

\begin{abstract}
This work explicates the physical basis of the material motions in living systems. Simplistic thinking sticks to an obvious seemingly unyielding scheme: nervous system sends control signals while the energy comes from the food. Such a trivial scheme is on par with robotics systems design supplementing artificial muscles with an extraneous supply of electrical energy. But how in living systems can energy appear immediately at a given place and time in suitable quantities? That comes from the nervous system activities utilizing moving excitations rather than simply sending ordinary electrical signals. In this case, neural signaling actually generates energy employing neural pulses relocations and a new physical resource for energy in association with the Cellular Automaton Universe. Organisms cannot simply generate mechanical work from the heat of the metabolism, as revealed by the so called Exercise Paradox. A Universe without an external influx of energy could not support life and may come to the ultimate equilibrium of the so called "heat death". Whereas the Physical World with a continual influx of mechanical energy to living mater may be subjected to periodic Global Warming incited by particular changes in Earth's biomass. In individual organisms a similar effect appears as recurrent fever attacks.
\end{abstract}

Keywords: Muscular Energy; Neuristor; Cellular Automaton Universe; Global Warming; Exercise Paradox

\section{Introduction}

\section{Statement of the problem: energy actions in the living systems}

The famous book [1] by Albert Szent-Gyorgyi delves into one of the most basic problems of biology: How does energy drive life? How does it move the living machine? [1] And there is no answer still. It is indicated there: difficulty in approaching the problem of energy transformation in muscle is misled by the formalism of our thermodynamic book keeping using utterly the law of conservation of energy. So, making up the energy balance sheets of reactions we usually express both the potential energy of a bond and the kinetic forms of energy in calories and thus, unconsciously, accept their identity. Gyorgyi AS had shown that contraction in muscle is essentially, the interaction of actomyosin with ATP and ions. Of the two proteins, myosin is responsible for the elementary act of contraction and so we can ask how the energy of P moves myosin, and hence the muscle? It is said in [1] that we do not know how muscle contracts, how it uses bond energy to produce work. "And the more we know about its structure the less we understand its function. We might have arrived here at the edge of the chasm which seems to extend through medicine and biology." Energy per sec does not produce work. To produce the requisite work, a sequence of the energy holding ATP is to be applied, and thus, for the muscles operations it is necessary to have special motor-generators supplying continual streams of carriers of the required energy. A brief outline of this idea has been presented in our abstract [2]. Also, the conceptual prospect for such a scheme in some way has been foreseen [3]. Namely, what we have suggested is that the energy for the motility of living objects is to be created employing unexpected resources from the effect of neural pulse propagation. Ordinarily, this effect is considered only for information signals transmissions. For this unusual suggestion, we rely on the fundamental problems of energy that come out in view of our Cellular Automaton model of the Physical Universe [4,5].

\section{The baffling purport of the transmission of signals by neural pulses}

One of the hottest subjects that my colleagues and I have vigorously discussed when I just started my career in superconductor Microelectronics [6] was the problem: why control signals in human brain and body have to be transmitted by the propagating excitations in neural fibers, rather than by electrical current directly? The riddle is: do we need complicated neuromorphic signals to attain the apparent performance of biological systems, while the control information can be just transmitted by plain electric wires much more effectively. Additional regulations, if necessary, like changing 
synapses weights, can be supplemented plainly, as e.g., in the socalled neural networks. So, it appears that neural organization might have some deeper commission beyond neuromorphing processing of information. In case, the significance of neural type propagations might become apparent in some unforeseeable future, some kind of these activities has been introduced into consideration with superconductor devices [7].

The figure 1 presents the suggested superconductor neuristor imitating neural pulse propagation.

The given scheme can be seen as a sequence of short superconductive segments shunted by normal conductor pieces. In the limiting case this scheme could become a continuous superconductive wire, like Sn, covered by a normal metal like $\mathrm{Cu}$. The electrical current, I, applied to this construction goes first to the superconductor segment having zero resistance, when the superconductive state of the segment with this current is destroyed and transforms it into a normal state with higher resistance, then the electric current is pushed out to a less resistant shunt, so the superconductor segment cools down and the current returns back to the zero resistance position; meanwhile the normal phase segment of the superconductor spreads due to heating to the neighboring segment that switches it to a normal phase, and these activities are repeated. As a result, a narrow region of normal phase will run along this wire structure. The suggested transmission of excitations in the presented superconductor neuristor is analogous to the propagation of electrochemical patterns in the nerve pulses. In figure 2 a neural propagation scheme is shown and we can see there the following analogies.

1. An active region in the superconductor neuristor is determined by heat in normal phase segment, analogously to positive electrical charges in the nerve.

2. A refractory region in the superconductor neuristor is determined by the transient process of heat fading away analogously to vanishing of electrical charges.

Beyond the delivery of information signals neuristors functionality has a vital significance for the restoration of disordered energy (heat or chemical bonds) into well-organized useful energy. The directional relocations of neuronal contents are determinative for concentrating the otherwise disordered energy of heat and chemical wanderings into mechanical energy for fixed purposeful motions. Organizations of such transformations for the superconductor neuristor and ordinary neural machinery are illustrated in figures 3 and 4 .

Figure 3 shows how an applied thermal pulse in the course of its propagation goes inside of electric coils inducing electrical current by changing magnetic field due to the so called Meissner effect [8]. The impact of this electric current can be stored in the accumulator for future purposes. This consideration is purely for conceptual deliberations.

Figure 4 shows that in the course of neural pulse propagation the energy are accumulated in the storage capacity of the Adenosine $5^{\prime}$-Triphosphate (ATP), which is a ubiquitous substance in the central and peripheral nervous systems. ATP is generated from ADP.

The question is where muscles get well-shaped mechanical energy for purposeful motions. The metabolic energy supplied with food in a disordered form of heat and is largely consumed for chemical activities in organs, like brain and liver. The well-shaped mechanical energy is produced using ATP molecules that serve as "the principal immediate donors of free energy rather than as a storage form of free energy". Typically, an ATP molecule is consumed within a minute following its formation. "Thus, during strenuous exertion, the rate of utilization of ATP may be as high as $0.5 \mathrm{~kg}$ per minute." [9].

It should be strictly emphasized that the energy containing ATP is actually created from the energy deficient ADP with the ordered motion of the neural pulse. And the ATP is immediately delivered to muscles by nervous system exactly to the place where the neural signal is going. It is very important to comprehend that, the creation of the ATP requires well-organized energy, and that apparently chaotic metabolic calories are not suitable for this task. So, to gain their motility, living objects must rely on certain action energy, different from heat. To this end we must consider an extraneous source of kinetic energy in the Universe; such source of energy naturally appears in connection with our surmised machinery of the Cellular Automaton Universe [4,5].

The presented novel source of energy empowers motions of living matter. Also, it implies the vital consequences of Global Warming. These issues are succinctly discussed in the conclusion. Thus, we are planning a more precise specification of our model of the Cellular Automaton Universe. The distinctive advantage of this model is that it clearly exposes the otherwise murky conception of energy [5]. So, before future theoretical elaboration let us consider here a recent experimental discovery of the Kinematical Anisotropy of the Universe $[10,11]$ that conclusively supports our envisioned origin of the mechanical energy.

\section{On the kinematical anisotropy of the universe}

We have considered the experimental and theoretical investigations of Dai J $[10,11]$ devoted to the anisotropy of the Brownian Motion.

The point is that, in general, any kind of a Cellular Automaton construction of the Physical Universe incorporates an inherent distinctive direction for the motion of all generated material formations as a whole. Such a construction should impose anisotropy on the motion of the molecules. So, this circumstance could be detected in the anisotropy of Brownian motion. Looking for the suspected effect on the Internet I came across the indicated papers [10,11]. Markedly, in these works the observed global anisotropy of the universe is also associated with the origination of energy.

Thus, Brownian motion should exhibit an axial symmetry around the direction of relocations of the material formations in the Cellular Automaton Universe. Notably, according to my theoretical considerations the material formations undergo relocations towards Virgo Cluster [12]. So, we anticipate that the suspected anisotropy of the Brownian motion should exhibit an axial symmetry around the direction to Virgo cluster. In some respect, this anticipation is in a certain agreement with the experimental data [10], which show some similarities in the diffusion distribution of round shape patterns of granules for spring an autumn data. This is in concordance with the direction towards Virgo, which is aligned with the Sun as a Zodiac Constellation of September. As to the differences determined by the varying directions arising from of the motion of Earth in spring

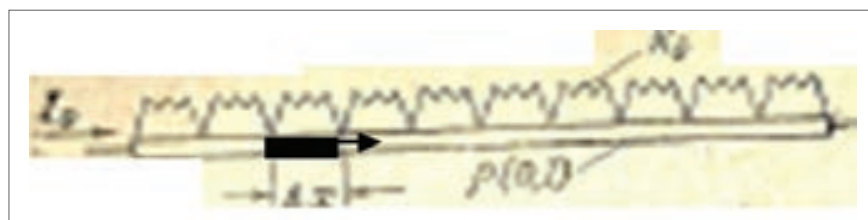

Figure 1: Imitation of electro-chemical neural transmissions with the propagation of normal regions in a superconductor neuristor. 


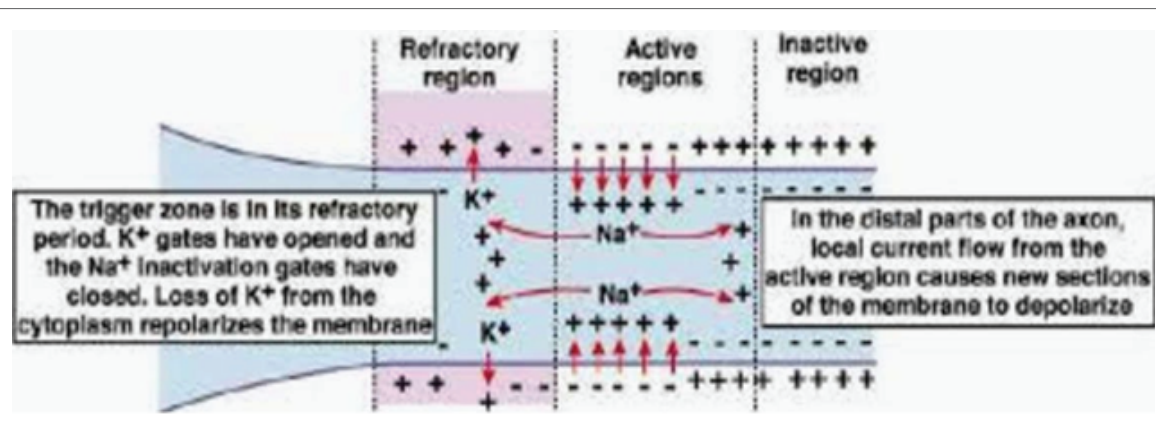

Figure 2: The scheme of nerve pulse propagation.

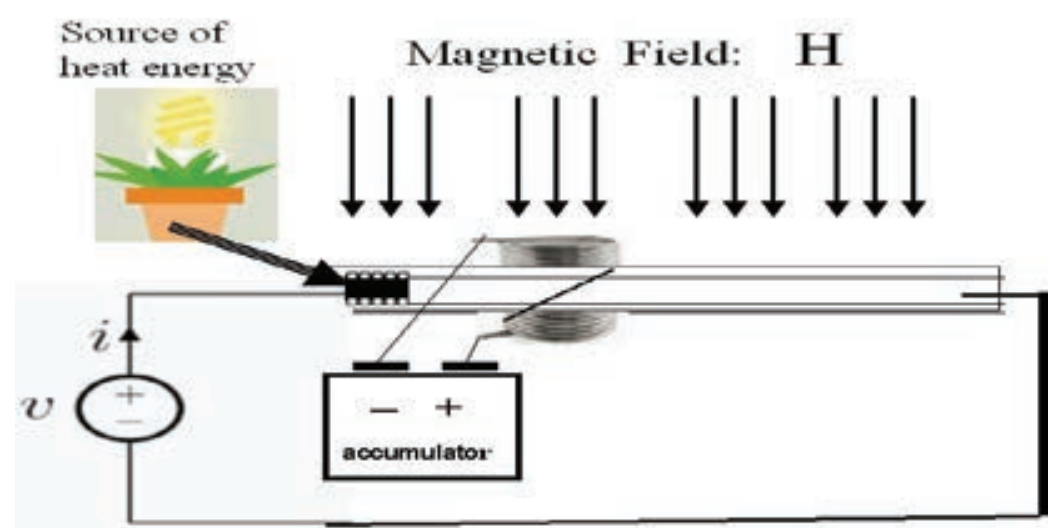

Figure 3: Transformation of the forced propagation of heat pulses into purposeful electro-mechanical energy.

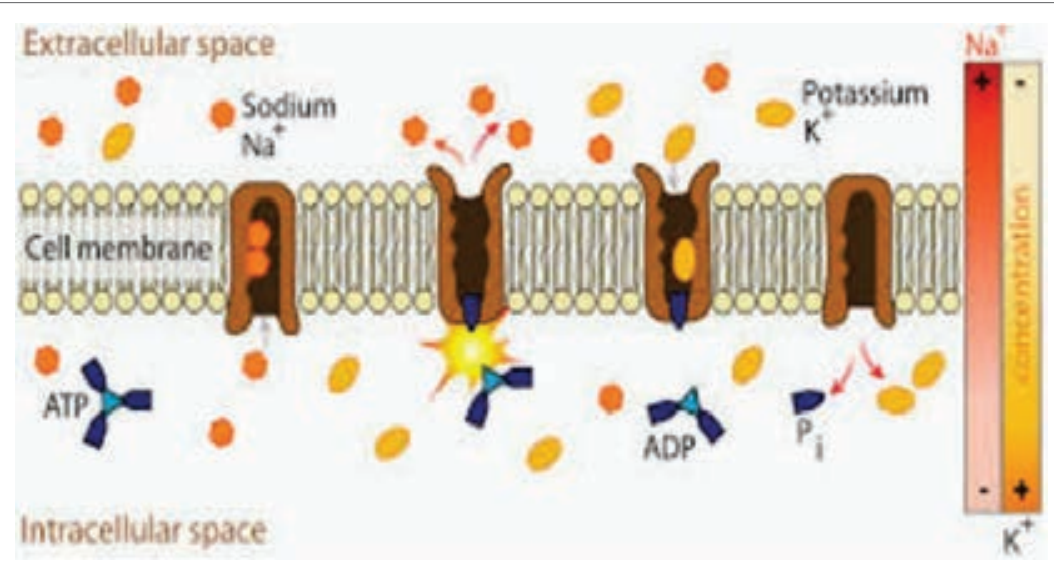

Figure 4: Stimulated formation of ATP and its delivery to muscles for extraction of mechanical energy

and autumn they are not very influential since the orbital velocity of the Earth $(\sim 30 \mathrm{~km} / \mathrm{sec})$ is by on order of magnitude smaller than the solar velocity towards Virgo $(\sim 600 \mathrm{~km} / \mathrm{sec})$. Thus, we get the following result: as long as a core of a molecular compound is moving to the prevalent direction towards Virgo Cluster the edge components are dragged in this prevalent direction, and as a result acquire some extra kinetic energy with respect to the main frame of reference. This extra kinetic energy can be used as a supplementary adding up to particular molecular transformations, like ADP into ATP. In other type of transformations, these activities just will end up in creation of more heat.
As to non-organic matter, like tectonic plates, the indicated effect results in misalignments of bulk bodies aggregates accumulated elastic energy that will be released as earthquakes. Presumably, most components of tectonic plates have predominant orientation for their cellular automaton generative motion in the main direction of the Virgo Cluster. Therefore, in the month of September there will be less strong misalignments for accumulation of elastic energy, and hence less chances for strong earthquakes [5] considering these problems and referencing to the table given in the figure 5.

\section{Discussion and Conclusion}




\begin{tabular}{|c|c|c|}
\hline \multicolumn{3}{|c|}{$\begin{array}{l}\text { World's Twelve Largest Earthquakes } \\
\text { Includes all measured earthquakes since } 1900\end{array}$} \\
\hline Magnitude & Location & Date \\
\hline 9.5 & Chile & $05 / 22 / 1960$ \\
\hline 9.2 & Alaska & $03 / 28 / 1964$ \\
\hline 9.1 & Off the coast of Northern Sumatra & $12 / 26 / 2004$ \\
\hline 9.1 & Honshu, Japan & $03 / 11 / 2011$ \\
\hline 9.0 & Kamchatka & $11 / 04 / 1952$ \\
\hline 8.8 & Off the coast of Chile & $02 / 27 / 2010$ \\
\hline 8.8 & Off the coast of Ecuador & $01 / 31 / 1906$ \\
\hline 8.7 & Rat Islands, Alaska & $02 / 04 / 1965$ \\
\hline 8.6 & Tibet & $08 / 15 / 1950$ \\
\hline 8.6 & Off the coast of Northern Sumatra & $04 / 11 / 2012$ \\
\hline 8.6 & Northern Sumatra & $03 / 28 / 2005$ \\
\hline 8.6 & Andreanof Islands, Alaska & $03 / 09 / 1957$ \\
\hline \multicolumn{3}{|c|}{ Data from the United States Geological Survey. } \\
\hline
\end{tabular}

Figure 5: A prediction of ultimate clarity: no strongest earthquakes (>8.5) in the month of September.

\section{The "Exercise paradox" extension}

An evolutionary anthropologist at Duke University Pontzer H had discovered a very puzzling effect: the so called Exercise Paradox [13]. Surprisingly, exercise isn't the route to weight loss you thought it was $[14,15]$. "The idea that activity dictates how many calories you burn is now naïve" "How can it be that people do more exercise without seeming to expend more energy?" This embarrassing observation is directly resolved with our suggested source of extraneous energy that is readily consumed by biological objects. Furthermore, this situation naturally extends as well to what can be called a "Fever Paradox": "Where can a starving person get heat energy for a fever without calories from food?"

\section{Global warming as a fever attack of the super organism of earth and a routine medical approach to its treatment}

The discovery of the all-pervading influx of energy to the physical Universe due to its Cellular Automaton mechanism reveals the ecological calamity of Global Warning as a sort of a Fever Paradox for the entire organism of Earth. The organism of Earth contains an abundance of Biomass in different forms of Life from groups of primitive creatures to rich diversity of multicellular plants and animals $[16,17]$. This Biosphere spreads over the Earth's surface, penetrates inside the crust, and goes into the depth of the World Ocean. The idea that all energy for the whole totality of the biological objects on Earth originates from the Sun is deficient. In accordance with our suggestion all the living organisms positively consume mechanical energy for their motions. Finally, it transforms into heat. As a result the Biosphere of the Earth warms up, or so to say gets "Fever". Therefore, the organism of Earth gets fever from certain taxons may be, even from some bacteria. Actually, bacteria are the second major biomass component, constituting $\approx 15 \%$ of the Global biomass [16]. For the Fever to go away the given taxon is to eradicate. A common Fever extending over an individual organism could totally destroy and kill this organism. In the case of the Global Warming Fever some parts of Earth's biosphere could survive, so the progression of Life may prolong in a crippled form. To avoid such situation we have try to follow a routine medical approach to combat the Fever of Global Warming. Thus, first we have to undertake "laboratory" tests to determine what taxa are mostly involved in its origination; those may even be some micro organisms, like bacteria, as indicated above. Second, we can try to discover a kind of a special substance, like "penicillin" that may eradicate the culprit taxa. Search for the biological material that allegedly implicate global warming fever may be undertaken by massive probes of biosphere samples, especially from subterranean organisms, and also from marine biota, testing them for possible spontaneous raises of their temperatures in isolated conditions.

As long as our concept regarding the external influx of mechanical energy for living matter is correct the suggested method of revealing the culprit biological objects with their possible suppression would present the only way to actually contain the Global Warming. Other measures could not discontinue this natural ecological effect of self-oscillations. Optimistically, the selfoscillations of the Global Warming Fever could come to an end in this manner by themselves as happens somehow in spontaneous healings of ordinary fever.

\section{References}

1. Gyorgyi AS (1957) Academic Press, Inc New York.

2. Berkovich S (2018) Nervous system as the producer of pinpoint energy for motions of living organisms. J Cell Sci Ther 9: 49.

3. Muscle loss in old age linked to fewer nerve signals: BBC News.

4. Berkovich S (2015) Law of inertia and the primal energy in the cellular automaton universe. J Eng C Mech 2: 62-67.

5. Berkovich S (2019) On the origin of kinetic energy in the Physical Universe including the Godforsaken area of turbulence. B05.00014: 1-35. 
6. Likharev KK (2012) Superconductor digital electronics. Physica C: Superconductivity and its Applications 482: 6-18.

7. Berkovich S Ya (1966) Superconductor Model of Nerve Impulse Behavior. In: Radio Engineering and Electronic Physics, Scripta Publishing Company, Cornell University, USA 293-295.

8. Meisner Effect.

9. Stryer L (1981) Biochemistry. $2^{\text {nd }}$ Edition, WH Freeman and Company, San Francisco, USA.

10. Dai J (2014) Macrosopic anisotropic Brownian motion is related to the directional movement of a "Universe field." Nat Sci 6: 54-58.

11. Dai J (2012) "Universe collapse model" and its roles in the unification of four fundamental forces and the origin and the evolution of the universe. Nat Sci 4: 54-58.

12. Berkovich S (2005) Prediction of the Virgo axis anisotropy: CMB radiation illuminates the nature of things 1-41.

13. Pontzer H (2017) The Exercise Paradox. Scientific American 28-31.

14. Burrell T (2019) Calories in crisis. New Sci 241: 30-33.

15. Pontzer H (2019) Step on it. New Sci 242: 34-37.

16. Bar-On YM, Philips R, Milo R (2018) The biomass distribution on Earth. PNAS 115: 6506-6511.

17. Scharf GA (2017) The Zoomable Universe. Scientific American, New York, USA. 